Aim Embed three measures from the OACC suite into clinical practice in our service.

Method Phased implementation began with identification of IPOS champions for each service area. Champions received training on Phase of Illness (POI) and Australia-Modified Karnofsky Performance Status (AKPS). Email communication was sent to all staff regarding OACC. Posters were displayed and project team members were available to answer questions. POI and AKPS were then routinely recorded across the service.

A training package was devised and delivered to all staff on using IPOS with patients. Community teams implemented IPOS into clinical practice first, followed by the inpatient units.

Initial audit used a randomised purposive sampling approach to obtain a sample of $10 \%$ of the caseload over the initial three month period which included patients from all service areas. The patient records for these patients was then reviewed using a standardised proforma to determine whether IPOS had been considered and completed where appropriate.

Results $84 \%$ of patients had at least one IPOS considered. IPOS was not considered at only $36 \%$ of encounters.

IPOS is now completed weekly and reviewed in MDT for all inpatients. It is considered at each encounter for community patients. Responses are used to guide clinical decisionmaking.

Conclusions Appropriate use of IPOS was much higher in our implementation period than reported for other services (Bausewein, Schildmann, Rosenbruch, Haberland et al., 2018).

We attribute this success to the quality and structure of staff training, phased implementation and to the visibility of the project team throughout the implementation process (Antunes, Harding \& Higginson on behalf of EUROIMPACT, 2014).

\section{P-104 THE EVOLUTION OF IMPACT REPORTING IN A HOSPICE DAY SERVICE}

Katherine Rugen, Aimee Doyle, Kate Dreyer, Chris Crompton, Kate McIntegart, Kate Marley. Woodlands Hospice, Liverpool, UK

\subsection{6/bmjspcare-2019-HUKNC.127}

Existing quantitative evidence for the benefits of Hospice Day Services is limited. Clinical Commissioning Group funding decisions are often based on numbers of attendees, rather than overall impact on individuals engaging with those services.

At Woodlands Hospice, an outcome measures questionnaire, the Integrated Palliative Care Outcome Scale (iPOS), was implemented on the inpatient unit several years ago, and, in addition to being clinically useful for individual patients, audit data showed an overall improvement in average iPOS score during patients' admission.

The use of iPOS was piloted for new patients to our Day Services at their initial assessment from December 2018. This tool was used to assist in transforming our individual patient care plan, with a change in our practice being to repeat iPOS for each patient every four weeks to update the care plan. A baseline audit showed a mean overall improvement in patients' iPOS score when repeated after four weeks. Interventions during the four-week intervening period varied according to the patient's own personalised plan of care. The interventions ranged from solely medical outpatient review, to multidisciplinary assessment and management, with some patients attending individual outpatient sessions only, and others attending group therapy sessions.

This audit looks at the breakdown in improvement of physical symptoms and psychological wellbeing, and compares the reduction in iPOS score to changes in other outcome measures, namely the OACC Phase of illness and the Palliative Performance Scale. The use of these outcome measures will enable the hospice to monitor the effect of the care and interventions it provides to each individual patient and ensure their personal care plan is continually adapted in accordance with their specific needs as their condition changes.

\section{P-105 MANAGEMENT OF A TRAINEE-LED RESEARCH COLLABORATIVE}

${ }^{1}$ Heena Khiroya, ${ }^{1}$ Natasha Freeman, ${ }^{1}$ Hazel Coop, ${ }^{1,2}$ Clare Marlow, ${ }^{1,3,4}$ Derek Willis. ${ }^{1}$ WM CARES, Wolverhampton, UK; ${ }^{2}$ New Cross Hospital, The Royal Wolverhampton NHS Trust, Wolverhampton, UK; ${ }^{3}$ Severn Hospice, Telford, UK; ${ }^{4}$ University of Chester, Chester, UK

10.1136/bmjspcare-2019-HUKNC.128

Background The West Midlands Collaboration Actioning Research in End of life and Supportive Care (WM CARES) is a palliative medicine trainee-led research collaborative, established in 2016. WM CARES enables trainees to be involved in research to benefit patient care and to gain experience in research prior to becoming consultants (Coop, Macfarlane, Twigger, Doré et al., 2017).

Published literature reveals several experiences of setting up trainee-led research collaboratives (Chamberlain, Schofield, Hancock, Etkind et al., 2019; Dowswell, Bartlett, Futaba, Whisker et al., 2014) but there is a paucity of information relating to the ongoing management and output of these collaboratives. Existing published work relates mainly to surgical specialties (Dowswell, Bartlett, Futaba, Whisker et al., 2014; Bhangu, Kolias, Pinkney, Hall et al.,2013; Jamjoom, Phan, Hutchinson \& Kolias, 2016).

Aim This work aims to describe our experience of managing and maintaining a trainee-led research collaborative within the field of palliative medicine. We wanted to understand potential barriers to maintaining a registrar-led research collaborative.

Methods We distributed a survey to the palliative medicine registrars in the West Midlands deanery in March 2019 to explore past, present and future levels of engagement with WM CARES.

Results Fourteen trainees (78\% of total) completed the survey. To date, WM CARES has published two papers (Coop \& Marlow, 2019; Macfarlane, Shayler, Nelms, Willis et al., 2018) and the latter has sparked the idea for the first project being conducted by the UK Palliative trainees Research Collaborative (Chamberlain, Schofield, Hancock, Etkind et al., 2019). Despite the pool of trainees changing since the conception of WM CARES, there is willingness to take on leadership roles within the collaborative, with all roles except one expected to be filled next year. The most common reasons cited for not taking on roles were maternity leave $(40 \%)$ and 